\title{
On the bend number of circular-arc graphs as edge intersection graphs of paths on a grid
}

\author{
Liliana Alcón ${ }^{1,1}$, Flavia Bonomo ${ }^{1,1}$, Guillermo Durán ${ }^{1,1,1}$, \\ Marisa Gutierrez ${ }^{1,1}$, María Pía Mazzoleni ${ }^{1,1}$, Bernard Ries ${ }^{1}$, \\ Mario Valencia-Pabon ${ }^{1}$ \\ ${ }^{a}$ Dto. de Matemática, FCE-UNLP, La Plata, Argentina \\ ${ }^{b}$ Dto. de Computación FCEN-UBA, Buenos Aires, Argentina \\ ${ }^{c}$ Dto. de Matemática e Inst. de Cálculo FCEN-UBA, Buenos Aires, Argentina \\ ${ }^{d}$ Dto. de Ingeniería Industrial, FCFM-Univ. de Chile, Santiago, Chile \\ ${ }^{e}$ Université de Fribourg, DIUF, Fribourg, Switzerland \\ ${ }^{f}$ Université Paris-13, Sorbonne Paris Cité LIPN, CNRS UMR7030, Villetaneuse, \\ France. Currently in Délégation at the INRIA Nancy - Grand Est, France \\ ${ }^{g}$ CONICET
}

\begin{abstract}
Golumbic, Lipshteyn and Stern [? ] proved that every graph can be represented as the edge intersection graph of paths on a grid (EPG graph), i.e., one can associate with each vertex of the graph a nontrivial path on a rectangular grid such that two vertices are adjacent if and only if the corresponding paths share at least one edge of the grid. For a nonnegative integer $k, B_{k}$-EPG graphs are defined as EPG graphs admitting a model in which each path has at most $k$ bends. Circular-arc graphs are intersection graphs of open arcs of a circle. It is easy to see that every circular-arc graph is a $B_{4}$-EPG graph, by embedding the circle into a rectangle of the grid. In this paper, we prove that circular-arc graphs are $B_{3}-\mathrm{EPG}$, and that there exist circular-arc graphs which are not $B_{2}$-EPG. If we restrict ourselves to rectangular representations (i.e., the union of the paths used in the model is contained in the boundary of a rectangle of the grid), we obtain EPR (edge intersection of paths in a rectangle) representations. We may define $B_{k}$-EPR graphs, $k \geq 0$, the same way as $B_{k}$-EPG graphs. Circular-arc graphs are clearly $B_{4}$-EPR graphs and we will show that there exist circular-arc graphs that are not $B_{3}$-EPR graphs. We also show that normal circular-arc graphs
\end{abstract}

\footnotetext{
${ }^{1}$ Dedicated to Martin Charles Golumbic on the occasion of his 65th birthday
} 
are $B_{2}$-EPR graphs and that there exist normal circular-arc graphs that are not $B_{1}$-EPR graphs. Finally, we characterize $B_{1}$-EPR graphs by a family of minimal forbidden induced subgraphs, and show that they form a subclass of normal Helly circular-arc graphs.

\section{Keywords.}

Keywords: edge intersection graphs, paths on a grid, forbidden induced subgraphs, (normal, Helly) circular-arc graphs, powers of cycles 2000 MSC: 05C62, 05C75, 05C85

\section{Introduction}

Let $\mathcal{G}$ be a rectangular grid of size $(\ell+1) \times(\ell+1)$. The vertical grid lines will be referred to as columns and denoted by $x_{0}, x_{1}, \ldots, x_{\ell}$, and the horizontal grid lines will be referred to as rows and denoted by $y_{0}, y_{1}, \ldots, y_{\ell}$. A grid point lying on column $x$ and row $y$ is referred to as $(x, y)$. A path on $\mathcal{G}$ is nontrivial if it contains at least one edge of the grid. Let $\mathcal{P}$ be a collection of nontrivial simple paths on $\mathcal{G}$. The edge intersection graph of $\mathcal{P}$ (denoted by $\operatorname{EPG}(\mathcal{P})$ ) is the graph whose vertices correspond to the paths of $\mathcal{P}$ and two vertices are adjacent in $\operatorname{EPG}(\mathcal{P})$ if and only if the corresponding paths in $\mathcal{P}$ share at least one edge in $\mathcal{G}$. A graph $G$ is called an edge intersection graph of paths on a grid (EPG graph) if $G=\operatorname{EPG}(\mathcal{P})$ for some $\mathcal{P}$. Every graph $G$ satisfies $G=\operatorname{EPG}(\mathcal{P})$ for some $\mathcal{P}$ on a large enough grid and allowing an arbitrary number of bends (turns on a grid point) for each path [? ]. In recent years, the subclasses for which the number of bends of each path is bounded by some integer $k \geq 0$, known as $B_{k}-E P G$ graphs, were widely studied [? ? ? ? ? ? ]. The bend number of a graph $G$ (resp. a graph class $\mathcal{H})$, is the smallest integer $k \geq 0$ such that $G$ (resp. every graph in $\mathcal{H}$ ) is a $B_{k}$-EPG graph. We denote by $B_{k}$-EPG, $k \geq 0$, the class of $B_{k}$-EPG graphs.

In [?], it was shown that for every integer $k \geq 0$ there exists a graph with bend number $k$, and that recognizing $B_{1}$-EPG graphs is NP-complete. The bend number of classical graph classes was investigated as well. In [? ], it was shown that outerplanar graphs are $B_{2}$-EPG graphs and that planar graphs are $B_{4}$-EPG graphs. For planar graphs, it is still an open question whether their bend number is equal to 3 or 4 . On the other hand, it is easy to see that $B_{0}$-EPG graphs exactly correspond to interval graphs (i.e., intersection graphs of intervals on a line) [? ]. A generalization of interval graphs are 
circular-arc (CA) graphs, i.e., intersection graphs of open arcs on a circle. It is natural to see circular-arc graphs as EPG graphs by identifying the circle with a rectangle of the grid. Hence, circular-arc graphs form a subclass of $B_{4}$-EPG graphs. This leads to some natural questions. For example, the bend number of circular-arc graphs or the characterization of circular-arc graphs that are $B_{k}$-EPG graphs, for some $k<4$. One of the main results of this paper is that the bend number of circular-arc graphs is 3 .

Another interesting question is how many bends per path are needed for a circular-arc graph to be represented in a rectangle of the grid, i.e., in such a way that the union of the paths is contained in the boundary of a rectangle of the grid. We call such graphs edge intersection graphs of paths on a rectangle (EPR graphs). It is easy to see that EPR graphs are exactly the circular-arc graphs. We will study the classes $B_{k}$-EPR, for $0 \leq k \leq 4$, in which the paths on the grid that represent the vertices of the graph have at most $k$ bends. As before, we denote by $B_{k}$-EPR, $k \geq 0$, the class of $B_{k}$-EPR graphs. Similar to the case of EPG graphs, one can define for a circular-arc graph $G$ the bend number with respect to an EPR representation as the smallest integer $k$ such that $G$ is a $B_{k}$-EPR graph. Notice that CA $=\mathrm{EPR}=B_{4}$-EPR. We strengthen this observation by showing that the bend number for circulararc graphs with respect to EPR representations is 4 . Furthermore, we focus on $B_{1}$-EPR graphs and $B_{2}$-EPR graphs $\left(B_{0}\right.$-EPR graphs correspond again to interval graphs), and relate these classes with the class of normal Helly circular-arc graphs. In summary, we obtain the following results: we prove that the bend number of normal circular-arc graphs with respect to EPR representations is 2 ; moreover, we characterize $B_{1}$-EPR graphs by a family of minimal forbidden induced subgraphs, and show that they are exactly the normal Helly circular-arc graphs containing no powers of cycles $C_{4 k-1}^{k}$, with $k \geq 2$, as induced subgraphs.

An extended abstract of a preliminary version of this work was published in the proceedings of LAGOS 2015 [? ].

\section{Preliminaries}

All graphs that we consider in this paper are connected, finite and simple. For all graph theoretical terms and notations not defined here, we refer the reader to [?].

We denote by $C_{n}, n \geq 3$, the chordless cycle on $n$ vertices. A graph is called chordal, if every cycle of length at least four has a chord. Given a 
graph $G$ and an integer $k \geq 0$, the power graph $G^{k}$ has the same vertex set as $G$, two vertices being adjacent in $G^{k}$ if their distance in $G$ is at most $k$.

Let $G=(V, E)$ be a graph and let $X \subseteq V$. We denote by $G-X$ the subgraph of $G$ induced by the vertex set $V-X$.

A clique (resp. a stable set) is a subset of vertices that are pairwise adjacent (resp. nonadjacent). We say that a vertex $v$ dominates a vertex $w$ if they are adjacent and every neighbor of $w$ is also a neighbor of $v$.

A thick spider $S_{n}, n \geq 2$, is the graph whose $2 n$ vertices can be partitioned into a clique $K=\left\{c_{1}, \ldots, c_{n}\right\}$ and a stable set $S=\left\{s_{1}, \ldots, s_{n}\right\}$ such that, for $1 \leq i, j, \leq n, c_{i}$ is adjacent to $s_{j}$ if and only if $i \neq j$. Notice that $S_{n_{1}}$ is an induced subgraph of $S_{n_{2}}$ if $n_{1} \leq n_{2}$. (The name spider for graphs or graph classes has been used in the literature with different meanings. We follow the notation in [? ], in the particular case in which the head of the spider is empty.)

Given a circle $\mathcal{C}$ of length $\ell$, we can assign to vertices $s_{1}, \ldots, s_{n}$ of the thick spider $S_{n}$ a set of pairwise disjoint arcs of $\mathcal{C}$, each of them of length $\ell / n-2 \varepsilon$, and to vertices $c_{1}, \ldots, c_{n}$ of $S_{n}$ a set of arcs of $\mathcal{C}$ of length $(n-1) \ell / n+\varepsilon$ each (where $\varepsilon$ is a small enough real number), in such a way that the arc corresponding to $c_{i}$ is disjoint from the arc corresponding to $s_{i}$ and intersects every other arc corresponding to a vertex in $S$, for $i=1, \ldots, n$. Notice that since the length of each of the arcs corresponding to vertices in $K$ is greater than $\ell / 2$, they are pairwise intersecting. So, $S_{n}$ is a circular-arc graph, as we have described a circular-arc model for it.

More in general, if $G$ is a circular-arc graph, $\mathcal{C}$ denotes the corresponding circle, and $\mathcal{A}$ the corresponding set of open arcs, then $(\mathcal{A}, \mathcal{C})$ is called a circular-arc model of $G[?]$. A graph $G$ is a Helly circular-arc graph (HCA graph) [? ] if it is a circular-arc graph having a circular-arc model such that any subset of pairwise intersecting arcs has a common point on the circle. Such a model is called a Helly model. A circular-arc graph having a circular-arc model without two arcs covering the whole circle is called a normal circular-arc graph (NCA graph), and such a model is called a normal model. Circular-arc models that are at the same time normal and Helly are precisely those without three or less arcs covering the whole circle (see, for example, Theorem 1 in [? ]). A graph that admits such a model is called a normal Helly circular-arc graph (NHCA graph) [? ]. We will denote by NCA (resp. NHCA) the class of normal (resp. normal Helly) circular-arc graphs.

In [? ], the authors present a characterization of NHCA graphs by a family of minimal forbidden induced subgraphs. Recent surveys on circular- 
arc graphs are given in [? ? ]. A very recent characterization of circular-arc graphs by forbidden structures is presented in [? ].

\section{Circular-arc graphs as EPG graphs}

The main result in this section is that the bend number of circular-arc graphs with respect to EPG representations is equal to 3 . We first show that every circular-arc graph is a $B_{3}$-EPG graph (Theorem ??), then we exhibit an example of a graph in $B_{3}$-EPG $\backslash B_{2}$-EPG (Proposition ??). We present also in this section a family of NHCA graphs that are not in $B_{1}$-EPG (Lemmas ?? and ??). This family will play a central role in the characterization of $B_{1}$-EPR graphs in the next section.

Theorem 1. Every circular-arc graph is a $B_{3}-E P G$ graph.

Proof. Let $G$ be a circular-arc graph and let $(\mathcal{A}, \mathcal{C})$ be a circular-arc model of $G$. Without loss of generality, we may assume that the endpoints of the arcs are all distinct and we can number them clockwise in the circle from 1 to $2 n$ (with $n$ being the number of vertices of $G$ ). We also define a point 0 in the circle between $2 n$ and 1 (clockwise). The arc $(a, b), 1 \leq a, b \leq 2 n$, denotes the arc from endpoint $a$ to endpoint $b$ (clockwise). In particular, an arc $(a, b)$ contains point 0 of $\mathcal{C}$ if and only if $a>b$. Let $X$ be the set of vertices in $G$ corresponding to arcs containing point 0 of $\mathcal{C}$. Clearly, these vertices form a clique in $G$. Moreover, $G-X$ is an interval graph that can be represented on a line by taking, for each vertex, the interval $(a, b)$ defined by the endpoints of its corresponding arc, since $a<b$ for vertices in $G-X$. We will construct the following EPG representation of $G$ on a grid. For each vertex in $G-X$ corresponding to an arc $(a, b)$, assign the 3-bends path on the grid whose endpoints are $\left(x_{0}, y_{b}\right)$ and $\left(x_{b}, y_{0}\right)$ and whose bend points correspond to the grid points $\left(x_{0}, y_{a}\right),\left(x_{a}, y_{a}\right),\left(x_{a}, y_{0}\right)$. For each vertex of $X$ corresponding to an arc $(c, d)$ (in this case $c>d$ ), assign the 3-bends path on the grid whose endpoints are $\left(x_{0}, y_{0}\right)$ and $\left(x_{2 n}, y_{0}\right)$ and whose bend points correspond to the grid points $\left(x_{0}, y_{d}\right),\left(x_{c}, y_{d}\right),\left(x_{c}, y_{0}\right)$. Since all the endpoints of the $\operatorname{arcs}$ in $\mathcal{A}$ are different, the edge intersections of the paths are either on column $x_{0}$ or on row $y_{0}$ of the grid. Clearly, two paths corresponding to vertices of $G-X$ intersect if and only if the corresponding arcs intersect on $\mathcal{C}$. Two paths corresponding to vertices of $X$ intersect at least on the edge of the grid going from $(0,0)$ to $(0,1)$. The path corresponding to a vertex in $G-X$ with 
endpoints $(a, b)$ and the path corresponding to a vertex in $X$ with endpoints $(c, d)$ intersect if and only if either $d>a$ or $c<b$, and the same condition holds for the corresponding arcs in $\mathcal{C}$. Thus, we obtain a representation of $G$ as a $B_{3}$-EPG graph.

Combining Theorem ?? with the following result shows that the bend number of circular-arc graphs with respect to EPG representations is 3.

Proposition 2. The thick spider $S_{40}$ is in $B_{3}-E P G \backslash B_{2}-E P G$.

Proof. Since all thick spiders are circular-arc graphs, it follows from Theorem ?? that $S_{40}$ is a $B_{3}$-EPG graph.

Suppose there exists a $B_{2}$-EPG representation of $S_{40}$. Let us consider the path $\mathcal{P}_{c}$ corresponding to a vertex $c$ of the clique and the paths corresponding to its 39 neighbors in the stable set $S$. The path $\mathcal{P}_{c}$ uses at most three lines (rows and/or columns) of the grid since it has at most 2 bends. Thus, $\mathcal{P}_{c}$ intersects at least 13 paths, $\mathcal{P}_{1}, \ldots, \mathcal{P}_{13}$, corresponding to 13 of its neighbors in $S$ on a same line $x$. Without loss of generality, we may assume that $x$ is a column of the grid.

Notice that, since the paths have at most 2 bends, the edges of each path on a same row or column form a connected subpath (this does not hold for example in paths with four bends: the 4-bends path on the grid whose endpoints are $(0,0)$ and $(0,3)$ and whose bend points correspond to the grid points $(0,1),(1,1),(1,2)$, and $(0,2)$, as the disconnected segments $(0,0)-(0,1)$ and $(0,2)-(0,3)$ on column 0$)$.

Consider now the 13 connected subpaths on $x$ corresponding to the paths $\mathcal{P}_{1}, \ldots, \mathcal{P}_{13}$ that $\mathcal{P}_{c}$ intersects on column $x$. Since these paths correspond to vertices of $S$, they are edge-disjoint and thus their subpaths on $x$ can be ordered. We may assume then that $P_{1}, \ldots, P_{13}$, the subpaths of $\mathcal{P}_{1}, \ldots, \mathcal{P}_{13}$ on column $x$, are ordered by index from left to right. Let $s_{j}, j \in\{1, \ldots, 39\}$, be the vertex in $S$ corresponding to the path $\mathcal{P}_{7}$, i.e., $\mathcal{P}_{s_{j}}=\mathcal{P}_{7}$. The path $\mathcal{P}_{c_{j}}$, corresponding to vertex $c_{j}$ of the clique that is not adjacent to $s_{j}$, cannot intersect the subpaths corresponding to $P_{1}, \ldots, P_{13}$ on both sides of $P_{7}$ on $x$ since it has at most two bends. Thus, it intersects at least 6 of them on some other row or column.

So we may assume, without loss of generality, that it intersects the paths $\mathcal{P}_{8}, \ldots, \mathcal{P}_{13}$ on some other row or column. But since these paths have at most two bends, are edge-disjoint and all use column $x$, it follows that $\mathcal{P}_{c_{j}}$ intersects 
at most 2 paths among $\mathcal{P}_{8}, \ldots, \mathcal{P}_{13}$ on a row. Therefore $\mathcal{P}_{c_{j}}$ necessarily uses two columns and one row, and it intersects at least 4 paths among $\mathcal{P}_{8}, \ldots, \mathcal{P}_{13}$ on a column $x^{\prime} \neq x$.

Since these 4 paths use both columns $x, x^{\prime}$ and they are edge disjoint, it follows that each of them uses a different row. Also notice that the order of their corresponding subpaths on the two columns $x, x^{\prime}$ from left to right must be the same, since they have at most 2 bends and therefore they cannot swap the order without intersecting.

Let $s_{k}, k \in\{1, \ldots, 39\}$, be the vertex corresponding to one of the two paths of these four whose subpaths on column $x^{\prime}$ are located in the middle (i.e., the second or third subpath). It is now easy to see that, given that fixed configuration for the four paths, it is impossible for the path $\mathcal{P}_{c_{k}}$, corresponding to vertex $c_{k}$ of the clique that is not adjacent to $s_{k}$, to avoid the path $\mathcal{P}_{s_{k}}$ while intersecting the paths corresponding to the remaining three vertices using only two bends. Thus $S_{40}$ does not admit a $B_{2}$-EPG representation.

The thick spider $S_{40}$ is probably not a minimal example in $B_{3}$-EPG $B_{2}$-EPG. The size 40 was chosen for the easy of presentation. Finding a characterization of the minimal graphs in (CA $\cap B_{3}$-EPG) $\backslash\left(\mathrm{CA} \cap B_{2^{-}}\right.$ $\mathrm{EPG})$ and in $\left(\mathrm{CA} \cap B_{2}\right.$-EPG $) \backslash\left(\mathrm{CA} \cap B_{1}\right.$-EPG) is left as an open problem.

We close this section by presenting a family of NHCA graphs that are not in $B_{1}$-EPG, and will be part of the characterization of $B_{1}$-EPR graphs in the next section: the powers of cycles $C_{4 k-1}^{k}$, with $k \geq 2$.

Lemma 3. Powers of cycles $C_{4 k-1}^{k}$, with $k \geq 2$, are $N H C A$.

Proof. Let $k \geq 2$ and let $G$ be the graph $C_{4 k-1}^{k}$, where the vertices of the cycle are denoted by $v_{1}, \ldots, v_{n}$, with $n=4 k-1$.

Let $\mathcal{C}$ be a circle of length $\ell$ and let $a_{1}, a_{2}, \ldots, a_{2 n}$ be $2 n$ points of it, ordered clockwise and such that the clockwise distance from $a_{i}$ to $a_{i+1}$ is $\ell /(2 n)$. Let $\mathcal{A}_{i}$, for $1 \leq i \leq n$, be the clockwise open arc from $a_{2 i-1}$ to $a_{2 i+2 k}$, where the index operations are performed modulo $2 n$. Let $\mathcal{A}=\left\{\mathcal{A}_{1}, \ldots, \mathcal{A}_{n}\right\}$. We will show first that $(\mathcal{A}, \mathcal{C})$ is a circular-arc model for $G$, with $\mathcal{A}_{i}$ corresponding to $v_{i}$. Indeed, as the length of all the arcs is the same and their start points are distinct, no arc is contained in another. Then the $\operatorname{arc} \mathcal{A}_{i}$ intersects exactly the arcs that have one of their endpoints within $\mathcal{A}_{i}$, which are, by definition of the arcs, $\mathcal{A}_{i+1}, \ldots, \mathcal{A}_{i+k}$ and $\mathcal{A}_{i-1}, \ldots, \mathcal{A}_{i-k}$.

We will show now that $(\mathcal{A}, \mathcal{C})$ is a normal Helly model. As we already noticed, all the arcs have the same length and, by definition, this length is 
$(2 k+1) \ell /(2 n)$. Also, the intersection of two arcs is either empty or of length at least $\ell /(2 n)$. So, with three $\operatorname{arcs} a, b$, and $c$ such that $a$ intersects $b$ and $c$ intersects $b$, we can cover at most a length of $3(2 k+1) \ell /(2 n)-2 \ell /(2 n)=$ $(6 k+1) \ell /(2 n)$. Observe that $(6 k+1) \ell /(2 n)<\ell \Leftrightarrow(6 k+1) \ell<2 n \ell$ and recalling that $n=4 k-1$, this reads $(6 k+1) \ell<(8 k-2) \ell$ which holds if and only if $2 k>3$, that is true for $k \geq 2$. As circular-arc models that are at the same time normal and Helly are precisely those without three or less arcs covering the whole circle, this completes the proof.

In order to prove the next result we need to introduce some more definitions. It was shown in [?] that an induced cycle $C_{4}$ in a graph $G$ corresponds to either a true pie or a false pie or a frame in any $B_{1}$-EPG representation of $G$ (see Figure ??). In a true pie or a false pie, the paths representing the vertices of the induced $C_{4}$ use one common grid point which is defined as the center of the pie. A frame is a model of $C_{4}$ such that each of the four corresponding paths has a bend in one of the four corners of a rectangle of the grid. Some examples of frame models are shown in Figure ??.

Lemma 4. Powers of cycles $C_{4 k-1}^{k}$, with $k \geq 2$, are not in $B_{1}-E P G$.

Proof. Let $k \geq 2$ and let $G$ be the graph $C_{4 k-1}^{k}$, where the vertices of the cycle are denoted by $v_{1}, \ldots, v_{n}$, with $n=4 k-1$. Suppose, by contradiction, that $\mathrm{G}$ admits a $B_{1}$-EPG representation. Note that $v_{1}, v_{k+1}, v_{2 k+1}$ and $v_{3 k+1}$ induce a $C_{4}$ in $G$.

We will consider the possible representations of the 4-cycle induced by $v_{1}, v_{k+1}, v_{2 k+1}$ and $v_{3 k+1}$. Let us denote by $\mathcal{P}_{i}$ the path corresponding to $v_{i}$, for $i=1, \ldots, n$. We will inductively show that, for each of the possible representations, the path $\mathcal{P}_{i+1}$ has to share with $\mathcal{P}_{i}$ a special point $p_{i}$ and its two incident edges of the grid. The point $p_{i}$ will be either the bend of $\mathcal{P}_{i}$ or the center of the pie in case of pie representations.

Suppose first that the cycle is represented by a true pie using column $x$ and row $y$ of the grid. Let $p$ be the intersection point of $x$ and $y$. Vertex $v_{2}$ is adjacent to $v_{1}, v_{k+1}$, and $v_{3 k+1}$ in $G$. Since its corresponding path $\mathcal{P}_{2}$ must intersect $\mathcal{P}_{1}, \mathcal{P}_{k+1}$ and $\mathcal{P}_{3 k+1}$ and it cannot intersect $\mathcal{P}_{2 k+1}$, it is forced to have a bend at $p$ and use the same semi-row and semi-column as $\mathcal{P}_{1}$. The same argument can then be applied to $v_{k+2}$ and $\mathcal{P}_{k+2}$ with respect to $\mathcal{P}_{k+1}$, to $v_{2 k+2}$ and $\mathcal{P}_{2 k+2}$ with respect to $\mathcal{P}_{2 k+1}$ and to $v_{3 k+2}$ and $\mathcal{P}_{3 k+2}$ with respect to $\mathcal{P}_{3 k+1}$. Considering now the cycle $v_{2}, v_{k+2}, v_{2 k+2}$ and $v_{3 k+2}$, we can repeat 


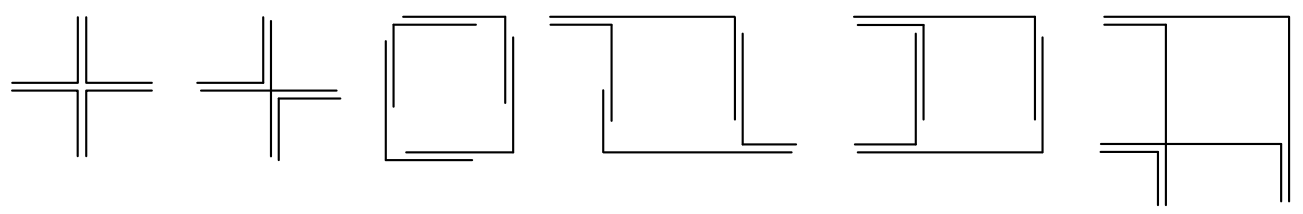

Figure 1: From left to right: a true pie, a false pie and four examples of a frame [? ].

the process and, after $k-1$ iterations, we will reach a contradiction because $\mathcal{P}_{1}$ should not use the same semi-row and semi-column as $\mathcal{P}_{3 k+1}$.

Suppose now that the 4-cycle is represented by a false pie with center $p=(x, y)$. By symmetry, we may assume that $\mathcal{P}_{1}$ has a bend at $p$ and $\mathcal{P}_{k+1}$ uses edges on column $x$ on both sides of $p$. Clearly, no path with at most one bend uses edges on two different rows or columns. Since $v_{2}$ is adjacent to $v_{3 k+1}, v_{1}$, and $v_{k+1}$, the path $\mathcal{P}_{2}$ must have a bend at $p$ and use the same semirow and semi-column as $\mathcal{P}_{1}$. A similar argument shows that $\mathcal{P}_{k+2}$ must use edges on column $x$ on both sides of $p$, as $\mathcal{P}_{k+1}$. Symmetrically, an analogous situation holds for $\mathcal{P}_{2 k+2}$ with respect to $\mathcal{P}_{2 k+1}$ and for $\mathcal{P}_{3 k+2}$ with respect to $\mathcal{P}_{3 k+1}$. As above, we can repeat the process and, after $k-1$ iterations, we will reach again a contradiction.

Finally, suppose that the 4-cycle is represented by a frame on the rectangle defined by columns $x$ and $x^{\prime}$ and rows $y$ and $y^{\prime}$. Suppose that $\mathcal{P}_{1}$ has edges on $x$ and $y, \mathcal{P}_{k+1}$ on $y$ and $x^{\prime}, \mathcal{P}_{2 k+1}$ on $x^{\prime}$ and $y^{\prime}$, and $\mathcal{P}_{3 k+1}$ on $y^{\prime}$ and $x$. In order to have edge intersections with $\mathcal{P}_{1}, \mathcal{P}_{k+1}$, and $\mathcal{P}_{3 k+1}$, the only possible row-column combination for the path $\mathcal{P}_{2}$ is to use edges on $x$ and $y$. Now repeating similar iterative arguments as previously, we will reach again a contradiction.

\section{Circular-arc graphs as EPR graphs}

In this section, we focus on representations of circular-arc graphs as edge intersection graphs of paths on the boundary of a rectangle of the grid, i.e., we restrict ourselves to 2 rows and 2 columns of the grid. Obviously, $\mathrm{CA}=$ $B_{4}$-EPR since we can embed the CA model into a rectangle of the grid in a natural way, and conversely. A strengthening of this observation is that the bend number of CA graphs with respect to EPR representations is equal to 
4. To show this, we exhibit in Proposition ?? a circular arc-graph that is not $B_{3}$-EPR.

In Theorem ?? we show that every normal circular-arc graph is a $B_{2}$-EPR graph, and we give a counterexample for the converse in Proposition ??.

In Lemma ?? we show that every graph in $B_{1}$-EPR admits a normal Helly circular-arc model. This property allows us to exhibit an example of a normal circular-arc graph that is not $B_{1}$-EPR, concluding that the bend number of NCA graphs with respect to EPR representations is equal to 2 . We also point out that there are graphs in $B_{1}$-EPG $\backslash B_{1}$-EPR.

In Theorem ??, we prove the equivalence of five statements for $B_{1}$-EPR graphs that are not chordal, two of them using structural properties, other two in terms of their NHCA models, and one in terms of forbidden induced subgraphs. Joining this result with known results from the literature on chordal NHCA graphs, we state in Theorem ?? a characterization of general $B_{1}$-EPR graphs. We prove that $B_{1}$-EPG $\cap \mathrm{NHCA}=B_{1}$-EPR $=\mathrm{NHCA} \cap$ $\left\{C_{4 k-1}^{k}\right\}_{k \geq 2}$-free. As NHCA graphs are characterized by minimal forbidden induced subgraphs [? ], this result implies a forbidden induced subgraph characterization of $B_{1}$-EPR graphs.

Finally, we sketch a linear-time algorithm to recognize $B_{1}$-EPR graphs.

Proposition 5. The thick spider $S_{13}$ is not in $B_{3}-E P R$.

Proof. By contradiction, suppose that $S_{13}$ admits a $B_{3}$-EPR representation. Clearly, at most four of the paths corresponding to vertices in the stable set contain a corner of the rectangle, since they are pairwise non adjacent. So, from the remaining 9 paths, representing vertices in the stable set, at least three of them are intervals completely contained in one side of the rectangle. Let us denote these paths in order by $\mathcal{P}_{i}, \mathcal{P}_{j}, \mathcal{P}_{k}$, representing vertices $s_{i}, s_{j}, s_{k}$. The path corresponding to vertex $c_{j}$ in the clique has to intersect $\mathcal{P}_{i}$, and $\mathcal{P}_{k}$ avoiding $\mathcal{P}_{j}$, so it necessarily needs four bends, a contradiction.

The thick spider $S_{13}$ is a minimal forbidden induced subgraph for the class of $B_{3}$-EPR graphs. We sketch, in Figure ??, $B_{3}$-EPR representations of $S_{13}-\left\{s_{1}\right\}$ and $S_{13}-\left\{c_{1}\right\}$ which, by symmetry, imply the minimality.

We will now consider normal circular-arc graphs (graphs having a circulararc model without two arcs covering the whole circle) and show that their bend number with respect to EPR representations is equal to 2 .

Theorem 6. Every NCA graph is a $B_{2}$-EPR graph. 

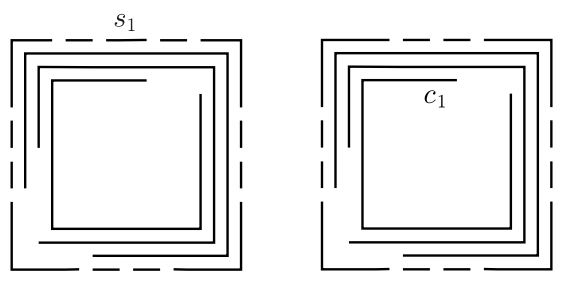

Figure 2: Sketches of $B_{3}$-EPR representations of $S_{13}-\left\{s_{1}\right\}$ and $S_{13}-\left\{c_{1}\right\}$ (we draw all the vertices of the stable set and representative vertices of the clique; other vertices in the clique can be represented symmetrically).

Proof. Let $(\mathcal{A}, \mathcal{C})$ be a NCA model of a normal circular-arc graph. Without loss of generality, we may assume that the endpoints of the arcs are pairwise different. Let $p$ be a point of $\mathcal{C}$ that is not the endpoint of an arc of $\mathcal{A}$. Since the model is normal, the union of the $\operatorname{arcs}$ of $\mathcal{A}$ that contain $p$ does not cover $\mathcal{C}$. Thus, there exists a point $q$ in $\mathcal{C}$ that is not the endpoint of an $\operatorname{arc}$ of $\mathcal{A}$ and is not contained in the union of the $\operatorname{arcs}$ of $\mathcal{A}$ containing $p$. We can then embed our model on a rectangle of the grid in the following way (arcs will bijectively correspond to paths and $\mathcal{C}$ will bijectively correspond to the rectangle): two consecutive corners of the rectangle correspond to point $p$ of the circle and the remaining two corners correspond to point $q$ of the circle. In this way, since no arc of $\mathcal{A}$ contains both $p$ and $q$, paths corresponding to arcs containing either $p$ or $q$ have two bends, while paths corresponding to arcs containing neither $p$ nor $q$ have no bend.

The converse is not true. We will show next that there exist $B_{2}$-EPR graphs that are not in NCA.

Proposition 7. The thick spider $S_{6}$ is in $B_{2}-E P R \backslash N C A$.

Proof. Let $(\mathcal{A}, \mathcal{C})$ be a circular-arc model of $S_{6}$. Without loss of generality, we may assume that the disjoint $\operatorname{arcs} \mathcal{A}_{1}, \ldots, \mathcal{A}_{6}$ representing the vertices $s_{1}, \ldots, s_{6}$ in the stable set are in clockwise order. The arc representing vertex $c_{1}$ intersects $\mathcal{A}_{2} \ldots, \mathcal{A}_{6}$ and is disjoint from $\mathcal{A}_{1}$, so it properly contains $\mathcal{A}_{3}$, $\mathcal{A}_{4}$, and $\mathcal{A}_{5}$. Similarly, the arc representing vertex $c_{4}$ properly contains $\mathcal{A}_{6}$, $\mathcal{A}_{1}$, and $\mathcal{A}_{2}$, and intersects $\mathcal{A}_{3}$ and $\mathcal{A}_{5}$, without intersecting $\mathcal{A}_{4}$. Thus the arcs representing vertices $c_{1}$ and $c_{4}$ cover the circle, and since the model was 

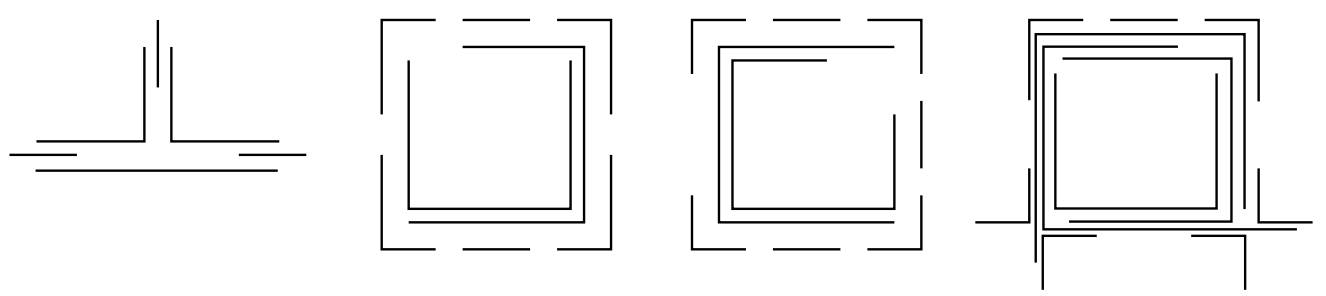

Figure 3: From left to right: a $B_{1}$-EPG representation of $S_{3}$; a sketch of a $B_{2}$-EPR representation of $S_{6}$ (we draw all the vertices of the stable set and representative vertices of the clique; other vertices in the clique can be represented symmetrically); sketches of $B_{3}$-EPR and $B_{2}$-EPG representations of $S_{7}$ (again, we draw all the vertices of the stable set and representative vertices of the clique; other vertices in the clique can be represented symmetrically).

arbitrary up to symmetries, $S_{6}$ is not in NCA. A $B_{2}$-EPR representation of $S_{6}$ is given in Figure ??.

We will now focus on $B_{1}$-EPR graphs and show that they are NHCA graphs.

Lemma 8. $B_{1}-E P R \subseteq N H C A$.

Proof. Consider a $B_{1}$-EPR representation of a graph $G$ and let $\mathcal{P}$ be the set of paths corresponding to the vertices of $G$. We will consider the natural bijection between the rectangle $\mathcal{R}$ and a circle $\mathcal{C}$, that maps the paths in $\mathcal{P}$ to open $\operatorname{arcs} \mathcal{A}$ of $\mathcal{C}$. Notice that two open arcs intersect if and only if the corresponding paths of $\mathcal{P}$ intersect on an least one edge of the grid. Thus, $(\mathcal{A}, \mathcal{C})$ is a circular-arc representation of $G$. Now, since each path has at most one bend and the arcs are open, the union of three (resp. two) arcs of $\mathcal{A}$ contains at most three (resp. two) points of $\mathcal{C}$ corresponding to corners of $\mathcal{R}$. Hence $(\mathcal{A}, \mathcal{C})$ is a NHCA model for $G$.

As a corollary, we obtain that the bend number of NCA graphs with respect to EPR representations is equal to 2. We know from Theorem ?? that it is at most 2. Now consider the thick spider $S_{3}$, which is in NCA (see for example [? ]). In [? ] it is shown that $S_{3}$ is not in NHCA, so by Lemma ??, it is not in $B_{1}$-EPR. 
The thick spider $S_{3}$ is also an example of a graph in $B_{1}$-EPG $\backslash B_{1}$-EPR, as it was shown in [?] that it belongs to $B_{1}$-EPG.

In the next theorem, we will introduce five equivalent statements for $B_{1^{-}}$ EPR graphs that are not chordal, two of them using structural properties, other two in terms of their NHCA models, and one in terms of forbidden induced subgraphs.

Theorem 9. Let $G=(V, E)$ be a graph which is not chordal. Then the following statements are equivalent:

(i) $G \in B_{1}-E P R$;

(ii) $G \in N H C A$ and $G$ contains no $C_{4 k-1}^{k}$, with $k \geq 2$, as induced subgraph;

(iii) $G \in N H C A$ and admits a NHCA model $(\mathcal{A}, \mathcal{C})$ with the following property: there are four points of $\mathcal{C}$, different from the endpoints of the arcs of $\mathcal{A}$, such that no arc of $\mathcal{A}$ contains two of these points;

$\left(\right.$ iii' $\left.{ }^{\prime}\right) \in N H C A$ and in every NHCA model $(\mathcal{A}, \mathcal{C})$ of $G$ there are four points of $\mathcal{C}$, different from the endpoints of the arcs of $\mathcal{A}$, such that no arc of $\mathcal{A}$ contains two of these points;

(iv) $G \in N H C A$ and $G$ has four disjoint connected subgraphs $H_{1}, H_{2}, H_{3}, H_{4}$, such that $H_{1}$ and $H_{3}$ are in different connected components of $G \backslash\left(V\left(H_{2}\right) \cup\right.$ $\left.V\left(H_{4}\right)\right)$ and $H_{2}$ and $H_{4}$ are in different connected components of $G \backslash\left(V\left(H_{1}\right) \cup\right.$ $\left.V\left(H_{3}\right)\right)$.

$\left(i v^{\prime}\right) G \in N H C A$ and $G$ has four disjoint complete subgraphs $H_{1}, H_{2}, H_{3}, H_{4}$, such that $H_{1}$ and $H_{3}$ are in different connected components of $G \backslash\left(V\left(H_{2}\right) \cup\right.$ $\left.V\left(H_{4}\right)\right)$ and $H_{2}$ and $H_{4}$ are in different connected components of $G \backslash\left(V\left(H_{1}\right) \cup\right.$ $\left.V\left(H_{3}\right)\right)$.

Proof. We will prove $(i i i) \Rightarrow\left(i v^{\prime}\right),(i v) \Rightarrow\left(i i i^{\prime}\right),(i) \Rightarrow(i i),(i i) \Rightarrow(i i i)$, $($ iii $) \Rightarrow(i)$. The implications $\left(i i i^{\prime}\right) \Rightarrow(i i i)$ and $\left(i v^{\prime}\right) \Rightarrow(i v)$ are straightforward.

$($ iii $) \Rightarrow\left(i v^{\prime}\right)$ : Let $(\mathcal{A}, \mathcal{C})$ be a circular-arc model of $G$ such that there exist four points $p_{1}, \ldots, p_{4}$ (clockwise) of $\mathcal{C}$ satisfying that no arc of $\mathcal{A}$ contains two of these points. Define $H_{i}$ as the subgraph induced by the vertices corresponding to $\operatorname{arcs}$ of $\mathcal{A}$ containing $p_{i}$, for $i=1, \ldots, 4$. Since $G$ is not chordal, the four graphs $H_{1}, \ldots, H_{4}$ are clearly non empty complete subgraphs, and since no arc contains two of the four points, they are disjoint. By the topology of the circle and the order of the points on it, it follows that every path connecting a vertex corresponding to an arc containing $p_{1}$ and 
a vertex corresponding to an arc containing $p_{3}$ necessarily contains either a vertex corresponding to an arc containing $p_{2}$ or a vertex corresponding to an arc containing $p_{4}$. So, $H_{1}$ and $H_{3}$ are in different connected components of $G \backslash\left(V\left(H_{2}\right) \cup V\left(H_{4}\right)\right)$ and, analogously, $H_{2}$ and $H_{4}$ are in different connected components of $G \backslash\left(V\left(H_{1}\right) \cup V\left(H_{3}\right)\right)$.

$(i v) \Rightarrow\left(i i i^{\prime}\right)$ : Let $H_{1}, H_{2}, H_{3}, H_{4}$ be disjoint connected subgraphs of $G$ such that $H_{1}$ and $H_{3}$ are in different connected components of $G \backslash\left(V\left(H_{2}\right) \cup\right.$ $\left.V\left(H_{4}\right)\right)$ and $H_{2}$ and $H_{4}$ are in different connected components of $G \backslash\left(V\left(H_{1}\right) \cup\right.$ $\left.V\left(H_{3}\right)\right)$. Let $(\mathcal{A}, \mathcal{C})$ be a NHCA model of $G$, and let $\mathcal{A}^{i}$ be the set of arcs corresponding to vertices of $H_{i}$, for $i=1, \ldots, 4$.

The union of the arcs in $\mathcal{A}^{i}$ induces a connected sector of $\mathcal{C}$, for each $i=1, \ldots, 4$. Moreover, by our assumptions, $\mathcal{A} \backslash\left(\mathcal{A}^{1} \cup \mathcal{A}^{3}\right)$ induces more than one connected sector on $\mathcal{C}$, such that arcs in $\mathcal{A}^{2}$ and in $\mathcal{A}^{4}$ are in different connected sectors $A_{2}$ and $A_{4}$. These sectors can be represented as two disjoint arcs of $\mathcal{C}$ with endpoints $t_{2}, h_{2}$, and $t_{4}, h_{4}$ (respectively) in clockwise order (the arc corresponding to $A_{i}$ is obtained by taking the union of all arcs in belonging to the same sector as $\mathcal{A}^{i}$, for $\left.i=2,4\right)$. Define $A_{1}$ and $A_{3}$ analogously, and let us represent them by two disjoint $\operatorname{arcs}$ of $\mathcal{C}$ with $t_{1}, h_{1}$, and $t_{3}, h_{3}$ being their endpoints, respectively. Notice that since the graph is not chordal, and thus $\mathcal{A}$ covers the circle, either $A_{1}$ covers $\left(h_{2}-\varepsilon, t_{4}+\varepsilon\right)$ and $A_{3}$ covers $\left(h_{4}-\varepsilon, t_{2}+\varepsilon\right)$, or $A_{1}$ covers $\left(t_{2}-\varepsilon, h_{4}+\varepsilon\right)$ and $A_{3}$ covers $\left(t_{4}-\varepsilon, h_{2}+\varepsilon\right)$, for some $\epsilon>0$. Without loss of generality, we may assume that the first case holds.

Let $p_{1}$ be a point in the clockwise open arc $\left(h_{2}, t_{4}\right), p_{2}$ in $\left(h_{1}, t_{3}\right), p_{3}$ in $\left(h_{4}, t_{2}\right)$, and $p_{4}$ in $\left(h_{3}, t_{1}\right)$. No arc of $\mathcal{A}^{1}, \mathcal{A}^{2}, \mathcal{A}^{3}$ or $\mathcal{A}^{4}$ contains two of these points, since these arcs are contained in $A_{1}, A_{2}, A_{3}$ and $A_{4}$, respectively. Furthermore, no arc in $\mathcal{A} \backslash \bigcup_{i=1}^{4} \mathcal{A}^{i}$ contains two of these points, because $A_{1}, A_{2}, A_{3}$ and $A_{4}$ are connected sectors of either $\mathcal{A} \backslash\left(\mathcal{A}^{1} \cup \mathcal{A}^{3}\right)$ or $\mathcal{A} \backslash\left(\mathcal{A}^{2} \cup \mathcal{A}^{4}\right)$.

At this point, we know $(i i i) \Rightarrow\left(i i i^{\prime}\right)$, and thus $(i i i) \Leftrightarrow\left(i i i^{\prime}\right)$.

$(i) \Rightarrow(i i)$ : Since $B_{1}$-EPR $\subseteq B_{1}$-EPG, this follows directly from Lemmas ??, ??, and ??.

$($ ii $) \Rightarrow($ iii $)$ : Let $G$ be a minimal counterexample to $(i i i)$, and let $(\mathcal{A}, \mathcal{C})$ be an arbitrary NHCA model of $G$. Then for every choice of four points of $\mathcal{C}$, there is an arc of $\mathcal{A}$ that contains two of these points. 
As usual, we will assume that the $\operatorname{arcs}$ in $\mathcal{A}$ are open and their endpoints are pairwise distinct. We can number the endpoints clockwise in the circle from 1 to $2 n$ ( $n$ being the number of vertices of $G$ ). For an $\operatorname{arc} \mathcal{A}_{i} \in \mathcal{A}$, its endpoints will be referred to as tail and head in such a way that $\mathcal{A}_{i}$ is the open arc traversing $\mathcal{C}$ clockwise from the tail to the head.

Claim 10. No arc of $\mathcal{A}$ is properly contained in another.

Proof of Claim ??. Suppose there is an arc $\mathcal{A}_{i}$ which is properly contained in an $\operatorname{arc} \mathcal{A}_{j}, i \neq j$. By minimality, and since $(i i i) \Rightarrow\left(i i i^{\prime}\right)$, the model $\left(\mathcal{A} \backslash\left\{\mathcal{A}_{i}\right\}, \mathcal{C}\right)$ admits four points $p_{1}, p_{2}, p_{3}, p_{4}$ such that no arc contains two of them. But if $\mathcal{A}_{j}$ does not contain two of these points, neither does $\mathcal{A}_{i}$ that is properly contained in $\mathcal{A}_{j}$. Thus, $(\mathcal{A}, \mathcal{C})$ satisfies the property as well, a contradiction to our hypothesis. $\diamond$

Claim 11. No vertex is dominated by another.

Proof of Claim ??. Suppose that vertex $v$ dominates vertex $w$. Let $\mathcal{A}_{v}$ and $\mathcal{A}_{w}$ be their corresponding arcs in the NHCA model $(\mathcal{A}, \mathcal{C})$. If there is an arc $\mathcal{A}_{z}$, corresponding to a vertex $z$, that intersects $\mathcal{A}_{w}$ only on $\mathcal{A}_{w} \backslash \mathcal{A}_{v}$, then, since $z$ is also adjacent to $v, \mathcal{A}_{z}$ intersects $\mathcal{A}_{v}$ only on $\mathcal{A}_{v} \backslash \mathcal{A}_{w}$. But then $\mathcal{A}_{v}, \mathcal{A}_{w}$ and $\mathcal{A}_{z}$ cover $\mathcal{C}$, a contradiction. Thus, such an arc of $\mathcal{A}_{z}$ does not exist. But then we can replace $\mathcal{A}_{w}$ by $\mathcal{A}_{w} \cap \mathcal{A}_{v}$ obtaining a NHCA model of the same graph with an arc properly contained in another, a contradiction with the previous claim. $\diamond$

It was shown by Golumbic and Hammer [? ] that the last claim implies that, when traversing the endpoints of the arcs on the circle clockwise, heads and tails necessarily alternate. Moreover, they proved that $G$ is the $t$-th power of the cycle $C_{n}$, for some value of $t$. Since $G$ is a counterexample to (iii), it follows that $t \geq 2$.

Let $1, \ldots, 2 n$ denote the endpoints of the arcs, where odd numbers correspond to tails and even numbers to heads. Thus, every arc is of the form $(2 i-1,2 i+2 t)$, for $i=1, \ldots, n$, where the sums are taken modulo $2 n$. In particular, every arc properly contains $2 t$ of the $2 n$ endpoints. Since the model is normal and Helly, $6 t<2 n$, otherwise arcs $(1,2 t+2),(2 t+1,4 t+2)$, and $(4 t+1,6 t+2)$ cover the circle. On the other hand, $8 t>2 n$, otherwise 
points $2,2 t+2,4 t+2$, and $6 t+2$ would be such that no arc of $\mathcal{A}$ contains two of them.

We will show now that $6 t<2 n<8 t$ is also a sufficient condition for the $t$-th power of the cycle $C_{n}$ to be a counterexample to $(i i i)$ (not necessarily minimal). It is clear that $6 t<2 n$ ensures that $C_{n}^{t}$ is a NHCA graph. Consider a NHCA model of $C_{n}^{t}$. Now, suppose $2 n<8 t$ and let $p_{1}, p_{2}, p_{3}, p_{4}$ be four points of $\mathcal{C}$. We may assume that they correspond to endpoints of arcs, otherwise we can move each of them to its closest endpoint without creating a new containment relation between arcs and points. If there are two points at distance at most $2 t-2$, i.e., in the closed interval $[i, i+2 t-2]$ for some $i=1, \ldots, n$, then they are both contained either in the arc $(i-1, i+2 t)$ or in the arc $(i-2, i+2 t-1)$, depending on the parity of $i$. So we may assume now that $p_{1}, p_{2}, p_{3}, p_{4}$ are pairwise at distance at least $2 t-1$. It follows from the inequality $2 n<8 t$ that at least two pairs of vertices are at distance exactly $2 t-1$ on $\mathcal{C}$, and at least one of these pairs corresponds to endpoints $i, i+2 t-1$ with $i$ even. Thus, these two points are both contained in the $\operatorname{arc}(i-1, i+2 t)$.

Since $t \geq 2$, the inequality $6 t<2 n$ implies that $n \geq 7$ thus, by the property above, $C_{7}^{2}$ is a minimal counterexample to $(i i i)$. Indeed, $C_{n}^{t}, t \geq 2$, contains $C_{7}^{2}$ as induced subgraph if and only if $12 t<4 n \leq 14 t$ (it can be verified that the $\operatorname{arcs}(1,2 t+2),(2 t+1,4 t+2),(4 t+1,6 t+2), \ldots$, $(12 t+1,14 t+2)$, where the operations are done modulo $2 n$, induce $\left.C_{7}^{2}\right)$.

More in general and inductively, we can prove that $C_{4 k-1}^{k}$, with $k \geq 2$, is a minimal NHCA counterexample to $($ iii $)$ and that $C_{n}^{t}, t \geq 2$, contains $C_{4 k-1}^{k}$ as induced subgraph if and only if $2(4 k-5) t<2(k-1) n$ and $2 k n \leq 2(4 k-1) t$, or equivalently, $(4 k-5) /(k-1)<n / t \leq(4 k-1) / k$.

As $(4 k-1) / k$ converges to 4 as $k$ tends to infinity, every $C_{n}^{t}$ with $t \geq 2$ and such that $3<n / t<4$ contains a power of a cycle $C_{4 k-1}^{k}$ as induced subgraph, for some $k \geq 2$, and this completes the proof.

$($ iii $) \Rightarrow(i)$ : Let $(\mathcal{A}, \mathcal{C})$ be a circular-arc model of $G$ such that there exist four points on $\mathcal{C}$ satisfying that no arc of $\mathcal{A}$ contains two of these points.

We will place the corners of the rectangle in those four points. Since the $\operatorname{arcs}$ in $\mathcal{A}$ are open and we are assuming, without loss of generality, that the endpoints of the arcs are pairwise distinct, we may assume as well that the four points are different from all the arc endpoints. It is easy to see then that we can find a big enough rectangle in the grid such that we can represent the arcs as paths in the grid, maintaining the order of their endpoints, and 
placing the four corners at the desired points, and this will give us a $B_{1}$-EPR representation of $G$.

Now we are able to prove the following.

Theorem 12. Let $G=(V, E)$ be a graph. Then $G \in B_{1}-E P R$ if and only if $G \in N H C A$ and $G$ has no $C_{4 k-1}^{k}, k \geq 2$, as induced subgraph.

Proof. One implication follows immediately from Lemmas ?? and ??, since the class is hereditary. For the converse, if $G$ is not a chordal graph, then the result follows from Theorem ??. Suppose now that $G$ is chordal. It is shown in [? ] that a chordal NHCA graph is indeed an interval graph, thus a $B_{0}$-EPR graph and, in particular, a $B_{1}$-EPR graph.

Since $C_{4 k-1}^{k}, k \geq 2$, is not in $B_{1}$-EPG (see Lemma ??), it follows that $B_{1^{-}}$ $\mathrm{EPG} \cap \mathrm{NHCA}=B_{1}$-EPR $=\mathrm{NHCA} \cap\left\{C_{4 k-1}^{k}\right\}_{k \geq 2}$-free. We leave as an open problem the characterization of the minimal graphs in $B_{4}$-EPR $\backslash B_{3}$-EPR and in $B_{3}$-EPR $\backslash B_{2}$-EPR.

\subsection{Linear time recognition of $B_{1}-E P R$ graphs}

We will now sketch a linear-time algorithm to recognize $B_{1}$-EPR graphs. The algorithm is based on the linear-time recognition algorithm of NHCA graphs [? ], that outputs a NHCA model of the graph if there is one, and property $\left(\right.$ iii $\left.^{\prime}\right)$ of Theorem ??.

Let $G$ be a NHCA graph that is not chordal, and let $(\mathcal{A}, \mathcal{C})$ be a normal Helly circular-arc model of it. Let $A=a_{1}, \ldots, a_{2 n}$ be the endpoints of the $\operatorname{arcs}$ in $\mathcal{A}$, in clockwise order.

Let $P=p_{1}, \ldots, p_{2 n}$ be points of the circle such that $p_{i}$ is between $a_{i}$ and $a_{i+1}$ for $i=1, \ldots, 2 n$ (index operations are performed modulo $2 n$ ). We will define a function $f: P \rightarrow P$. For each point in $P$, let $f(p)$ be the first point clockwise in $P$ such that there is no arc of $\mathcal{A}$ containing both $p$ and $f(p)$.

Notice that, since the model covers the circle, $f\left(p_{i}\right) \neq p_{i+1}$, for every $i$. Moreover, if the point $a_{i+1}$ is a tail, then $f\left(p_{i+1}\right)=f\left(p_{i}\right)$, and if the point $a_{i+1}$ is the head of an $\operatorname{arc} \mathcal{A}_{v}$ in $\mathcal{A}$, then either $\mathcal{A}_{v}$ contains $f\left(p_{i}\right)$ and $f\left(p_{i+1}\right)$ is the point right after the tail of $\mathcal{A}_{v}$, or $f\left(p_{i+1}\right)=f\left(p_{i}\right)$. We can therefore compute $f\left(p_{1}\right), \ldots, f\left(p_{2 n}\right)$ in linear time.

Now, observe that if points $q, q^{\prime}, q^{\prime \prime}, q^{\prime \prime \prime}$ (clockwise) in $P$ satisfy the property that no arc of $\mathcal{A}$ contains two of these points, then points $q, f(q), f^{2}(q), f^{3}(q)$ 
satisfy this property as well. It is worth noticing that, since the model is normal and Helly, for every point $q$ in $P$ the points $q, f(q), f^{2}(q), f^{3}(q)$ are in clockwise order.

By definition of $f$, in order to check the property, we only need to check that there is no arc containing both $q$ and $f^{3}(q)$. Equivalently, we need to check that either $f^{4}(q)=q$ or $f^{3}(q), f^{4}(q), q$ are in clockwise order.

The algorithm is then as follows. Given a graph $G$, we first apply the algorithm in [?], that runs in $O(n+m)$ time, where $n$ and $m$ are, respectively, the number of vertices an edges of $G$. If $G$ is an interval graph, the algorithm outputs an interval model of $G$, as it uses as a sub-algorithm one of the many available interval graphs recognition algorithms. In this case the graph is $B_{0^{-}}$ EPR. If, otherwise, $G$ is not chordal, the algorithm outputs a normal Helly circular-arc model $(\mathcal{A}, \mathcal{C})$ of it.

Let $A=a_{1}, \ldots, a_{2 n}$ be the endpoints of the $\operatorname{arcs}$ in $\mathcal{A}$, in clockwise order. We define the set $P=p_{1}, \ldots, p_{2 n}$ and compute $f\left(p_{1}\right), \ldots, f\left(p_{2 n}\right)$ in linear time. We can then check for every point $q$ in $P$ if either $f^{4}(q)=q$ or $f^{3}(q), f^{4}(q), q$ are in clockwise order. If the answer is affirmative for some point $q$, the graph $G$ is $B_{1}$-EPR by Theorem ?? and the observations above. Moreover, we can place the bends of the rectangle in the representation at $q, f(q), f^{2}(q)$, and $f^{3}(q)$. If the answer is negative for every point $q$, the graph $G$ is not $B_{1}$-EPR, by the observations above.

Since checking the property for each $q$ in $P$ can be done in constant time once we have precomputed $f(P)$, the last step of the algorithm takes linear time. The overall complexity of the algorithm is then $O(m+n)$, the complexity of the NHCA recognition algorithm.

\section{Further results}

The thick spider $S_{3}$ is one of the minimal forbidden induced subgraphs for the class NHCA [? ], but all the thick spiders are CA graphs and, by Theorem ??, $B_{3}$-EPG graphs. Thick spiders allow us to distinguish classes in the families $B_{k}$-EPR $(k \leq 4), B_{k}$-EPG $(k \leq 3)$, NHCA and NCA. In the previous sections we have proved that $S_{40}$ is in $B_{3}$-EPG $\backslash B_{2}$-EPG (Proposition ??), and that $S_{6}$ is in $B_{2}$-EPR $\backslash \mathrm{NCA}$ (see Proposition ?? and Figure ??). In this section we will prove that $S_{7}$ is in $B_{3}$-EPR $\backslash B_{2}$-EPR (Proposition ??), as well as in $B_{2}$-EPG $\backslash B_{1}$-EPG (Proposition ??).

Proposition 13. The thick spider $S_{7}$ is in $B_{3}-E P R \backslash B_{2}-E P R$. 
Proof. Figure 3 shows a $B_{3}$-EPR representation of $S_{7}$. Let us show now that $S_{7}$ is not a $B_{2}$-EPR graph. By contradiction, suppose that $S_{7}$ admits a $B_{2}$-EPR representation. Let us consider only the paths corresponding to vertices in the stable set. If there are three of them whose union is contained in two adjacent sides of the rectangle (or in one side of the rectangle), let us say in order $\mathcal{P}_{i}, \mathcal{P}_{j}, \mathcal{P}_{k}$, representing vertices $s_{i}, s_{j}, s_{k}$, respectively, then the path corresponding to vertex $c_{j}$ in the clique has to intersect $\mathcal{P}_{i}$ and $\mathcal{P}_{k}$ avoiding $\mathcal{P}_{j}$, and so it needs at least three bends, a contradiction. So, we may assume that this situation does not occur. Consider two opposite corners of the rectangle. If they are not covered by two different paths corresponding to the stable set, then there are at least three paths whose union is contained in two adjacent sides of the rectangle, a contradiction. So, we may assume that the four corners are covered by paths, corresponding to (at most) four vertices of the stable set. If no such path uses two corners, then, from the remaining three paths, there are two of them that are intervals contained in the same side or in adjacent sides of the rectangle. But then, their union together with one path on a corner are contained in two adjacent sides of the rectangle, a contradiction. If exactly one of the paths uses two corners (and one side of the rectangle), from the remaining four paths, there are two of them that are intervals contained in the same side of the rectangle, so as before we obtain a contradiction. Finally, if there are two paths using two corners each, and two sides of the rectangle, from the remaining five paths, there are three of them that are intervals contained in the same side of the rectangle, a contradiction. This completes the proof.

The thick spider $S_{7}$ is not a minimal forbidden induced subgraph for the class of $B_{2}$-EPR graphs, but it is minimal within the family of thick spiders. Indeed, with a similar proof we can show that $S_{7}-\left\{c_{1}\right\}$ is still in $B_{3}$-EPR $\backslash$ $B_{2}$-EPR, but $S_{6}$ is in $B_{2}$-EPR (see Figure ??).

Proposition 14. The thick spider $S_{7}$ is in $B_{2}-E P G \backslash B_{1}-E P G$.

Proof. Figure 3 shows a $B_{2}$-EPG representation of $S_{7}$. Let us show now that $S_{7}$ is not a $B_{1}$-EPG graph. By contradiction, suppose that $S_{7}$ admits a $B_{1}$-EPG representation. Let us consider the path $\mathcal{P}_{c}$ corresponding to a vertex $c$ of the clique and the paths corresponding to its 6 neighbors in the stable set $S$. The path $\mathcal{P}_{c}$ has edges on at most two lines (one row and one column) of the grid. Thus, it intersects at least 3 paths corresponding to 
neighbors in $S$ on a same line $x$. Without loss of generality, we may assume that $x$ corresponds to a column of the grid. Consider now the 3 intervals on column $x$ belonging to the 3 paths mentioned above. Since the corresponding vertices are pairwise non adjacent, they admit some order on column $x$, say $\mathcal{I}_{i}, \mathcal{I}_{j}, \mathcal{I}_{k}$, corresponding to vertices $s_{i}, s_{j}, s_{k}$, respectively. Having at most one bend each, the paths corresponding to $s_{i}$ and $s_{k}$ do not have edges in a common column (and they do not have edges in a column different from $x$, or more than one interval on $x$ ). So there is no way for the path $\mathcal{P}_{c_{j}}$ corresponding to the vertex $c_{j}$ of the clique that is not adjacent to $s_{j}$ of avoiding the interval $\mathcal{I}_{j}$ while intersecting the paths corresponding to $s_{i}$ and $s_{k}$ using only one bend.

In this case $S_{7}$ is not a minimal example. Indeed, $S_{4}$ is not in $B_{1}$-EPG, but the size 7 was chosen for the easy of presentation. Another similar example of a circular-arc graph being not in $B_{1}$-EPG is the 4-sun [? ].

\section{Conclusions and open questions}

In this paper we study the number of bends per path needed to represent the class of circular-arc graphs as edge intersection graphs of paths on a grid.

One of the main results is that $\mathrm{CA} \subseteq B_{3}$-EPG. We also show that (CA $\left.\cap B_{3}-\mathrm{EPG}\right) \backslash\left(\mathrm{CA} \cap B_{2}-\mathrm{EPG}\right)$ and $\left(\mathrm{CA} \cap B_{2}-\mathrm{EPG}\right) \backslash\left(\mathrm{CA} \cap B_{1}-\mathrm{EPG}\right)$ are non-empty, being the thick spiders $S_{40}$ and $S_{7}$, respectively, examples of graphs in these families.

These examples are not necessarily minimal, the sizes were chosen for the easy of presentation. Finding a characterization of the minimal graphs in $\left(\mathrm{CA} \cap B_{3}-\mathrm{EPG}\right) \backslash\left(\mathrm{CA} \cap B_{2}\right.$-EPG) and in $\left(\mathrm{CA} \cap B_{2}-\mathrm{EPG}\right) \backslash(\mathrm{CA} \cap$ $B_{1}$-EPG) is left as an open problem.

We also study a particular type of representation in which the union of the paths used in the model is contained in the boundary of a rectangle of the grid.

It is easy to see that $\mathrm{CA}=B_{4}$-EPR, and we prove that $B_{4}$-EPR $\backslash B_{3}$-EPR, $B_{3}$-EPR $\backslash B_{2}$-EPR, and $B_{2}$-EPR $\backslash B_{1}$-EPR are non-empty, being the thick spiders $S_{13}, S_{7}$, and $S_{3}$, respectively, examples of graphs in these families. Not all of them are minimal examples as graphs, but they are minimal as examples within thick spiders.

Concerning subclasses of circular-arc graphs, we prove that NCA $\subsetneq B_{2^{-}}$ $\mathrm{EPR}$. We also show that $\left(\mathrm{NCA} \cap B_{2}\right.$-EPR $) \backslash\left(\mathrm{NCA} \cap B_{1}\right.$-EPR) is non-empty, 
being the thick spider $S_{3}$ a minimal example in this family.

We prove that $B_{1}$-EPR $\subsetneq \mathrm{NHCA}$. Indeed, one of the main results in this paper is the equivalence $B_{1}$-EPG $\cap \mathrm{NHCA}=B_{1}$-EPR $=\mathrm{NHCA} \cap$ $\left\{C_{4 k-1}^{k}\right\}_{k \geq 2}$-free. As NHCA graphs are characterized by minimal forbidden induced subgraphs [? ], this result implies a forbidden induced subgraph characterization of $B_{1}$-EPR graphs.

We leave as an open problem the characterization of the minimal graphs in $B_{4}$-EPR $\backslash B_{3}$-EPR and in $B_{3}$-EPR $\backslash B_{2}$-EPR.

Finally, we sketch a linear-time algorithm to recognize $B_{1}$-EPR graphs.

Acknowledgements. We would like to thank Marty Golumbic whose lectures about EPG graphs have motivated this research, and to Jayme Szwarcfiter, who posed some of these questions at LAGOS'09. We would also like to thank the anonymous referees for their valuable suggestions that helped us to significantly improve the paper. This work was partially supported by UBACyT Grant 20020130100808BA, CONICET PIP 112-201201-00450CO and 122-01001-00310, and ANPCyT PICT-2015-2218, 2012-1324 and 20101970 (Argentina), FONDECyT Grant 1140787 and Millennium Science Institute "Complex Engineering Systems" (Chile), "Sorbonne Paris Cité" Project (France), and MathAmSud Project 13MATH-07 (Argentina-Brazil-ChileFrance).

\section{References}

[1] L. Alcón, F. Bonomo, G. Durán, M. Gutierrez, P. Mazzoleni, B. Ries, and M. Valencia-Pabon. On the bend number of circular-arc graphs as edge intersection graphs of paths on a grid. Electronic Notes in Discrete Mathematics, 50:249-254, 2015.

[2] A. Asinowski and B. Ries. Some properties of edge intersection graphs of single-bend paths on a grid. Discrete Mathematics, 312:427-440, 2012.

[3] A. Asinowski and A. Suk. Edge intersection graphs of systems of paths on a grid with a bounded number of bends. Discrete Applied Mathematics, 157:3174-3180, 2009.

[4] T. Biedl and M. Stern. On edge intersection graphs of $k$-bend paths in grids. Discrete Mathematics \& Theoretical Computer Science, 12(1):1$12,2010$. 
[5] J.A. Bondy and U.S.R. Murty. Graph Theory. Springer, New York, 2007.

[6] Y. Cao, L.N. Grippo, and M.D. Safe. Forbidden induced subgraphs of normal Helly circular-arc graphs: characterization and detection. Discrete Applied Mathematics, in press. doi:10.1016/j.dam.2015.08.023.

[7] G. Durán, L.N. Grippo, and M.D. Safe. Structural results on circulararc graphs and circle graphs: a survey and the main open problems. Discrete Applied Mathematics, 164:427-443, 2014.

[8] D. Epstein, M.C. Golumbic, and G. Morgenststern. Approximation algorithms for $B_{1}$-EPG graphs. Lecture Notes in Computer Science, 8037:328-340, 2013.

[9] M. Francis, P. Hell, and J. Stacho. Forbidden structure characterization of circular-arc graphs and a certifying recognition algorithm. In P. Indyk, editor, Proceedings of the 26th Annual ACM-SIAM Symposium on Discrete Algorithms, pages 1708-1727, San Diego, CA, 2015.

[10] F. Gavril. Algorithms on circular-arc graphs. Networks, 4:357-369, 1974.

[11] M.C. Golumbic and P.L. Hammer. Stability in circular-arc graphs. Journal of Algorithms, 9:314-320, 1988.

[12] M.C. Golumbic, M. Lipshteyn, and M. Stern. Edge intersection graphs of single bend paths on a grid. Networks, 54:130-138, 2009.

[13] M.C. Golumbic, M. Lipshteyn, and M. Stern. Single bend paths on a grid have strong Helly number 4. Networks, 62:161-163, 2013.

[14] D. Heldt, K. Knauer, and T. Ueckerdt. Edge-intersection graphs of grid paths: the bend-number. Discrete Applied Mathematics, 167:144-162, 2014.

[15] D. Heldt, K. Knauer, and T. Ueckerdt. On the bend-number of planar and outerplanar graphs. Discrete Applied Mathematics, 179:109-119, 2014 .

[16] B. Jamison and S. Olariu. A tree representation for $P_{4}$-sparse graphs. Discrete Applied Mathematics, 35:115-129, 1992. 
[17] M.C. Lin, F.J. Soulignac, and J.L. Szwracfiter. Normal Helly circular-arc graphs and its subclasses. Discrete Applied Mathematics, 161:1037-1059, 2013.

[18] M.C. Lin and J.L. Szwarcfiter. Characterizations and linear time recognition of Helly circular-arc graphs. Lecture Notes in Computer Science, 4112:73-82, 2006.

[19] M.C. Lin and J.L. Szwarcfiter. Characterizations and recognition of circular-arc graphs and subclasses: A survey. Discrete Mathematics, 309(18):5618-5635, 2009.

[20] A. Tucker. Characterizing circular-arc graphs. Bulletin of the American Mathematical Society, 76:1257-1260, 1970. 